\title{
Cuando todo no es suficiente
}

\section{When everything is not enough}

\author{
Jorge CÁrdenas Roldán • Bogotá, D.C. (Colombia)
}

\section{Introducción}

La púrpura trombocitopénica trombótica (PTT) es una entidad que se encuentra clasificada bajo las microangiopatías trombóticas (MAT) y se caracteriza por conteo plaquetario severamente disminuido y anemia hemolítica no autoinmune (1). Desde su descripción en 1924 por Moschcowitz esta entidad se ha distinguido por una elevada mortalidad y morbilidad las cuales han disminuido con los avances en el tratamiento: plasmaféresis y esteroides. No obstante, hasta en el $10 \%$ casos hay refractariedad a esta terapia lo que plantea un reto terapéutico para los médicos a cargo de estos pacientes. Se presenta un caso de PTT refractaria al manejo convencional y la estrategia terapéutica instaurada.

\section{Presentación del caso}

Mujer de 34 años, soltera, madre de un hijo, secretaria, sin antecedentes de importancia. Consulta por aparición de equimosis en extremidades y tronco de tres días de evolución asociado petequias en cuello y en miembros inferiores, niega hemorragia asociada. A la revisión por sistemas la paciente refiere que presenta deposiciones Bristol 7 desde hace cuatro días, no disentéricas y que ceden espontáneamente un día antes del ingreso. Así mismo, refiere coluria e ictericia y niega adenopatías, trombosis o retos hemostásicos previos. Signos vitales al ingreso con tensión arterial de 130/78 mmHg, frecuencia cardiaca de 82 latidos $/ \mathrm{min}$, frecuencia respiratoria de 19 respiraciones $/ \mathrm{min}$, afebril y con índice de masa corporal de $33.6 \mathrm{Kg} / \mathrm{m} 2$. Otros hallazgos al examen físico son la presencia de conjuntivas hipocrómicas, petequias en paladar blando, duro y en mucosa labial, ictericia, equimosis y petequias en miembros inferiores. Se toman paraclínicos los cuales demuestran trombocitopenia (10300) y anemia ( $\mathrm{Hb} 10.3 \mathrm{~g} / \mathrm{dL}$ ), hiperbilirrubinemia a expensas de la indirecta y tiempos de coagulación y función renal normales. En el contexto de una paciente joven con un cuadro agudo de lo que pareciera una anemia hemolítica se solicitó perfil de hemólisis el cual demuestra anemia hemolítica no inmune y frotis de sangre periférica (FSP) con cambios inespecíficos. El perfil de autoinmunidad con anti RO positivo únicamente y perfil infeccioso y hematológico negativos. Dado que no había diagnóstico aparente se solicitó nuevo frotis de sangre periférica (FSP) que demuestra la presencia de esquistocitos.

Con la sospecha de una MAT en curso se iniciaron sesiones de plasmaféresis en la Unidad de Cuidado Intensivo (UCI), pulsos de metilprednisolona (MTP) y a la par se tomaron niveles de actividad de ADAMTS 13 con resultado en $0 \%$. La respuesta plaquetaria en los primeros cinco días fue favorable pasando de un conteo de 10300 - 138 000. Empero, al sexto día descienden a pesar de la terapia por lo que se administra un segundo pulso de MTP. Sin evidenciar evolución adecuada con el tratamiento, a la décima sesión de plasmaféresis se administró vincristina (VIN) nuevamente sin respuesta por lo que se aplicó rituximab (RTX) igualmente infructuoso. Se continuaron las sesiones de plasmaféresis y se administró segunda dosis de VIN en conjunto con ciclosporina, segunda dosis de RTX y tres días de azatioprina (AZA) pero la evolución plaquetaria y clínica de la paciente empeoraron. Después de 21 sesiones de plasmaféresis la paciente presenta paro cardiorrespiratorio asociado a hemoptisis masiva y fallece 25 días después de la llegada a la UCI.

\section{Discusión}

El término microangiopatía trombótica es un término de patología en el cual se evidencia microtrombos diseminados ricos en plaquetas y oclusión de arteriolas y capilares. En
Dr. Jorge C. Cárdenas Roldán: Residente de Medicina Interna, Universidad del Rosario - Fundación Cardio Infantil - Instituto de Cardiología. Bogotá, D.C. (Colombia). Correspondencia: Dr. Jorge Cárdenas Roldán. Bogotá, D.C. (Colombia)

E-Mail: jorgec.cardenas@urosario.edu.co 
el ámbito clínico se utiliza como término para agrupar una serie de patologías que comparten estos hallazgos patológicos y se caracterizan por tener una incidencia y prevalencia muy baja así como una elevada tasa de mortalidad incluso con tratamiento (1). Las entidades que componen este grupo son: el síndrome hemolítico urémico, síndrome hemolítico urémico atípico y la PTT. Si bien estas entidades comparten un sustrato etiopatogénico similar hay diferencias clínicas que permiten una clara diferenciación de las mismas. En la PTT se encuentran anticuerpos contra, o en algunos casos, deficiencia de la enzima ADAMTS 13 la cual hidroliza el factor de Von Willebrand que está anclado al endotelio. Al encontrarse la actividad de esta enzima disminuida, el multímero de dicho factor reacciona con las plaquetas formando trombos en la microcirculación los cuales generan trombosis, isquemia y aumento de la hemólisis por aumento del shear stress o tensión cortante.

El cuadro clínico característico que se ha descrito desde 1924 por Moschcowitz y reafirmado en 1966 y 1978 por Schulman y Upshaw, se compone por una péntada conformada por fiebre, trombocitopenia, anemia hemolítica microangiopática, síntomas neurológicos e insuficiencia renal (1). Pueden ocurrir otras manifestaciones como dolor abdominal, disnea y sangrado. Desde la descripción de la enfermedad la mortalidad de esta patología se ha presentado hasta en $90 \%$ de los casos (2) y desde la introducción de la plasmaféresis como tratamiento ha disminuido hasta $15 \%$ (2). Este tratamiento se ha complementado con el uso de pulsos de esteroides con el fin de disminuir la producción de autoanticuerpos dirigidos contra la enzima ADAMTS 13. Dada la baja prevalencia e incidencia de esta enfermedad (prevalencia 2:10 000 e incidencia anual de tres por millón) (1), los estudios realizados son reportes de casos, y en el mejor de los casos estudios fase 2, los cuales se han realizado en los últimos años. Esto ha llevado a la publicación de guías de tratamiento en el 2012, en el 2014 y 2017 (3-5) con la recomendación grado 1 y nivel de evidencia, A para el uso de la plasmaféresis y B para los esteroides como terapia de primera línea. Sin embargo hasta en 10-40\% (6) de los casos, la PTT puede ser refractaria (Plaquetas menor a 50 000 después de cinco sesiones de plasmaféresis y esteroides) o presenta una recidiva (Plaquetas menor a 50000 después de tener más de 150000 ), situación que presenta un reto terapéutico para el médico ya que aumenta la mortalidad y la morbilidad y no se conoce con claridad cuál y como sería la terapia a seguir.

Para este caso se ejecutó una revisión sistemática para evaluar el número de publicaciones en PTT refractaria que reportarán el uso favorable de un procedimiento. Esta búsqueda se llevó a cabo desde 1963 hasta la fecha, encontrándose reportes desde 1990. Lo que se halló es que el medicamento con más reporte de uso favorable es el RTX, con 68 publicaciones desde 1990 seguido por VIN con 13 publicaciones y 10 publicaciones para la esplenectomía y ciclosporina A (Tabla 1.) Llama la atención que la mayoría de los estudios revisados para todos los medicamentos/procedimientos sean reportes de caso salvo algunos favoreciendo rituximab que son fase 2 , multicéntricos y abiertos. Estos estudios han sido incorporados en la última guía (3) la cual sugiere, al igual que los estudios primarios, que el RTX debe administrarse de manera temprana ante una recidiva o una PTT refractaria. En Colombia el uso de RTX está restringido por el INVIMA al tratamiento de ciertos linfomas o para la artritis reumatoide por lo que la reciente incorporación de MIPRES por parte del Ministerio de Salud no cubriría este tratamiento para esta patología. Esta situación a todas luces va en contra vía de la salud y de la calidad de vida de nuestros pacientes; es nuestra responsabilidad el poder brindar el tratamiento adecuado de una manera eficaz y oportuna para ellos. Para solventar esta circunstancia se podría solicitar ante el INVIMA que se incluya la PTT como indicación para el uso de RTX y de los otros medicamentos (bortezomib). Empero, quien debe elevar esta petición ante el INVIMA es la casa farmacéutica

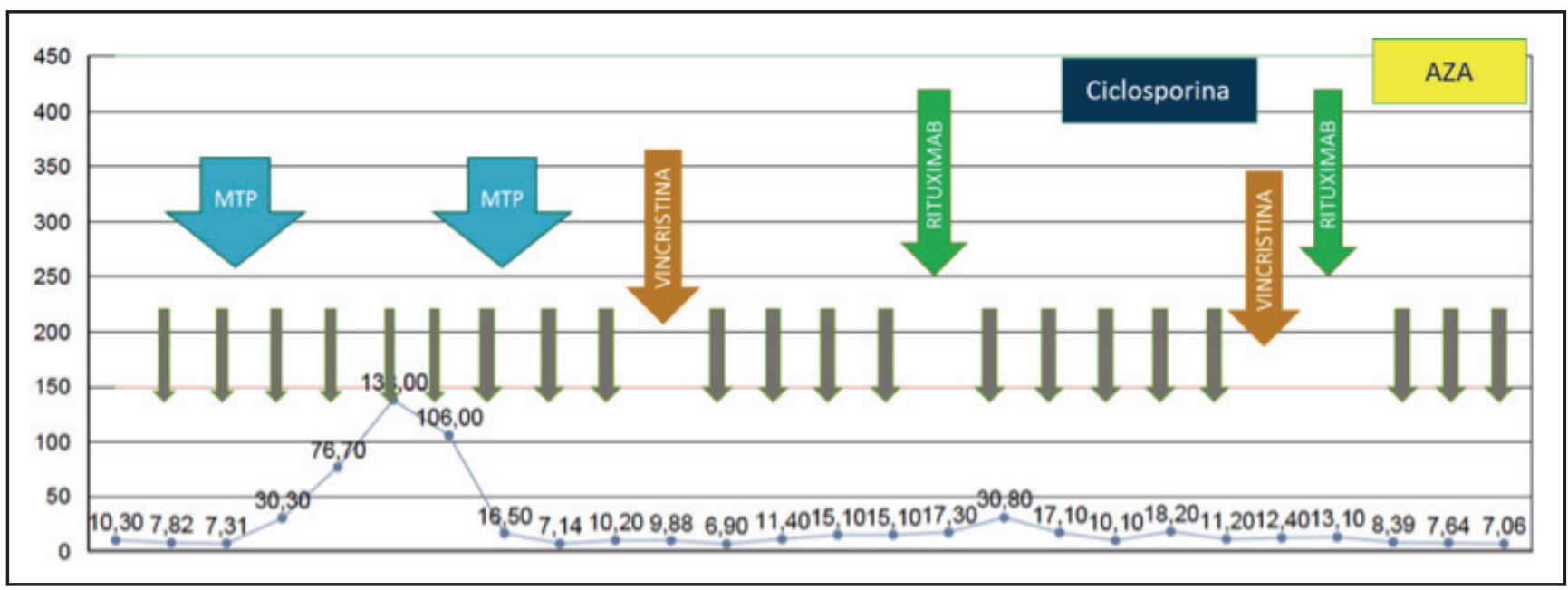

Figura 1. Evolución de conteo plaquetario y tratamiento administrado. (Cada punto de la curva indica un día. Las flechas grises indican sesión de plasmaféresis. AZA: azatioprina; MTP. metilprednisolona). 
Tabla 1. Número de publicaciones con uso favorable del tratamiento en PTT refractaria a partir de 1990.

\begin{tabular}{|c|c|c|c|c|c|c|c|c|c|}
\hline Año & Rituximab & Vincristina & Esplenectomia & Ciclosporina A & Bortezomib & Eculizumab & $\begin{array}{c}\text { Inmunoabsorción de } \\
\text { Proteina A } \\
\end{array}$ & Ciclofosfamida & NAC \\
\hline 1990 & 0 & 1 & 0 & 0 & 0 & 0 & 0 & 0 & 0 \\
\hline 1991 & 0 & 1 & 0 & 0 & 0 & 0 & 0 & 0 & 0 \\
\hline 1992 & 0 & 0 & 0 & 0 & 0 & 0 & 0 & 0 & 0 \\
\hline 1993 & 0 & 0 & 0 & 0 & 0 & 0 & 0 & 0 & 0 \\
\hline 1994 & 0 & 1 & 0 & 0 & 0 & 0 & 1 & 0 & 0 \\
\hline 1995 & 0 & 1 & 0 & 0 & 0 & 0 & 0 & 0 & 0 \\
\hline 1996 & 0 & 0 & 0 & 1 & 0 & 0 & 0 & 0 & 0 \\
\hline 1997 & 0 & 0 & 0 & 0 & 0 & 0 & 1 & 1 & 0 \\
\hline 1998 & 0 & 0 & 0 & 1 & 0 & 0 & 0 & 0 & 0 \\
\hline 1999 & 0 & 2 & 0 & 0 & 0 & 0 & 0 & 1 & 0 \\
\hline 2000 & 0 & 1 & 2 & 1 & 0 & 0 & 0 & 0 & 0 \\
\hline 2001 & 0 & 0 & 1 & 0 & 0 & 0 & 0 & 0 & 0 \\
\hline 2002 & 2 & 0 & 1 & 1 & 0 & 0 & 0 & 0 & 0 \\
\hline 2003 & 0 & 1 & 1 & 1 & 0 & 0 & 0 & 0 & 0 \\
\hline 2004 & 1 & 0 & 1 & 1 & 0 & 0 & 0 & 1 & 0 \\
\hline 2005 & 5 & 3 & 0 & 0 & 0 & 0 & 0 & 0 & 0 \\
\hline 2006 & 3 & 0 & 2 & 0 & 0 & 0 & 0 & 0 & 0 \\
\hline 2007 & 7 & 1 & 0 & 1 & 0 & 0 & 1 & 0 & 0 \\
\hline 2008 & 3 & 0 & 0 & 0 & 0 & 0 & 0 & 0 & 0 \\
\hline 2009 & 9 & 0 & 1 & 2 & 0 & 0 & 0 & 0 & 0 \\
\hline 2010 & 5 & 0 & 0 & 0 & 0 & 0 & 0 & 0 & 0 \\
\hline 2011 & 6 & 0 & 0 & 1 & 0 & 1 & 0 & 0 & 0 \\
\hline 2012 & 2 & 0 & 0 & 0 & 0 & 1 & 0 & 0 & 0 \\
\hline 2013 & 9 & 0 & 1 & 0 & 0 & 0 & 0 & 0 & 0 \\
\hline 2014 & 5 & 1 & 0 & 0 & 2 & 1 & 0 & 0 & 1 \\
\hline 2015 & 5 & 0 & 0 & 0 & 0 & 0 & 0 & 0 & 0 \\
\hline 2016 & 5 & 0 & 0 & 0 & 5 & 1 & 0 & 0 & 1 \\
\hline 2017 & 1 & 0 & 0 & 0 & 1 & 0 & 0 & 0 & 0 \\
\hline Total & 68 & 13 & 10 & 10 & 8 & 4 & 3 & 3 & 2 \\
\hline
\end{tabular}

que manufactura este medicamento y podría no estar dentro de sus intereses ya que la PTT es una enfermedad rara. Y es precisamente en su rareza que se puede plantear la solución y tiene que ver con un recurso olvidado por la mayoría de los internistas: las enfermedades huérfanas.

Según la Ley 1392 de 2010, las enfermedades huérfanas son aquellas que sean crónicamente debilitantes, graves, amenacen la vida y que tengan una prevalencia menor de 1:5000. Esta ley estipula (El Gobierno Nacional reconocerá de interés nacional las enfermedades huérfanas para garantizar el acceso a los servicios de salud y tratamiento y rehabilitación a las personas que se diagnostiquen con dichas enfermedades, con el fin de beneficiar efectivamente a esta población con los diferentes planes, programas y estrategias de intervención en salud, emitidas por el Ministerio de la Protección Social). Y siendo más específicos el artículo 8 de esta ley trata el tema de medicamentos (Con el fin de mejorar el acceso de los pacientes a los medicamentos huérfanos y una mejor administración de los recursos financieros, se faculta al Gobierno Nacional para... que permita el acceso equitativo para todos los pacientes). Según lo anterior las patologías incluidas al ser de interés nacional debe asegurarse el tratamiento y este será financiado por el FOSYGA.

El listado de enfermedades huérfanas se inició en 2013 y es dinámico, ya que es actualizado cada dos años por lo que se ha actualizado en el 2015 y en el 2017. Poniendo lo anterior en contexto, en caso de que la PTT fuera considerada una enfermedad huérfana el tratamiento no debería tener restricciones, pero a 2017 la PTT no se encuentra en esta lista.
¿Qué se puede hacer? Cualquier ciudadano colombiano puede someter a consideración del Ministerio de Salud la inclusión de una enfermedad que cumpla la definición de enfermedad huérfana y esto nos brindaría herramientas efectivas para el tratamiento oportuno de nuestros pacientes.

Con la presentación de este caso clínico se pretende mostrar la dificultad que puede presentar la PTT refractaria y una revisión de la literatura posiciona al RTX como una de las terapias más usadas y efectivas si es usado de manera temprana. Del mismo modo, se pretende redescubrir una herramienta terapéutica olvidada como lo son las enfermedades huérfanas y toda la legislación detrás de ellas ya que pudiera ser de provecho para el ejercicio médico de los internistas en pro del beneficio de nuestros pacientes.

\section{Referencias}

1. Arnold DM, Patriquin CJ, Nazy I. Thrombotic microangiopathies: a general approach to diagnosis and management. Canadian Medical Association Journal. 2016;189(4): 153--9.

2. Joly BS, Coppo P, Veyradier A. Thrombotic thrombocytopenic purpura. Blood 2017; 129(21): 2836-46.

3. Matsumoto M, Fujimura Y, Wada H, Kokame K, Miyakawa Y, Ueda Y, et al. Diagnostic and treatment guidelines for thrombotic thrombocytopenic purpura (TTP) 2017 in Japan. International journal of hematology. 2017.

4. Contreras E, de la Rubia Ja. Diagnostic and therapeutic guidelines of thrombotic microangiopathies of the Spanish Apheresis Group. Medicina clinica. 2015;144(7):331.e1--.e13.

5. Scully M, Hunt BJ, Benjamin S, Liesner R, Rose P, Peyvandi F, et al. Guidelines on the diagnosis and management of thrombotic thrombocytopenic purpura and other thrombotic microangiopathies. British Journal of Haematology. 2012; 158(3): 323--35.

6. Sayani FA, Abrams CS. How I treat refractory thrombotic thrombocytopenic purpura. Blood. 2015; 125(25): 3860-7. 